\title{
"A Proposed Revision of the Memorial Conference Classfication of Leprosy
}

\author{
II. W. Wade.
}

After discussing the various amendments to the Leonard Wood Memorial Conference classification of leprosy put forward by different writers, Dr. Wade suggests the following definition of terms and classification :-

Definitions.

In the present connection it is necessary to modify or extend certain of the definitions of the Memorial Conference classification in the light of present knowledge, and seems desirable to add certain others for the sake of completeness. The most important of the proposed modifications are the precision of "leproma" and the reduction of "leprotic" to its general sense. "Macule" is retained in its special sense, which leprosy workers will undoubtedly continue to give it in spite of the protests of dermatologists. The most important added definition is "lepride," which was unfortunately overlooked by the conference.

Leproma. This term applies exclusively to the lesions of various organs that are characteristic of the cutaneous type of leprosy. The condition is a granulomatous one in which reaction on the part of the invaded tissues is minimal. The essential histological feature is an accumulation of "lepra cells," which may show little differentiation from their original form (the macrophage), or may contain globi, or may have undergone multiple vacuolation to produce the so-called Virchow cells, of ten multinucleate. These cells contain leprosy bacilli in considerable and of ten great numbers, though they also occur in other cells. Lepromatous lesions in the skin may be so slight as to be imperceptible, ranging up to marked, extensive infiltrations or conspicuous nodular masses. As a rule they are more ill-defined and diffusely outlined than the leprides, and they do not exhibit the same tendency to radial extension or the same changes of color or sensation.

Lepride. This term is applied to the discrete macular lesions of the skin that are characteristic of neural leprosy. The leprides vary greatly in appearance, size, and as regards elevation; they may be flat, or even depressed (through atrophy), or markedly thickened; they may be smooth-surfaced or very irregular ("granular," "pebbled" or micropapulate); they tend to enlarge radially and to merge with adjacent ones, and to undergo central resolution. Diminution of sensory perception, partial or complete, is a typical feature, though its development may be delayed. The definitely infiltrated leprides, at least, are granulomatous, the essential feature being the nonspecific "tuberculoid" change, with which there usually is banal chronic inflammatory infiltration of variable degree. Associated cutaneous nerves may be

*Abstracted from the American Journal of Tropical Medicine, November, 
similarly affected and may undergo necrosis or even cold-abscess formation. These lesions result from the reaction of the tissue to the presence of the leprosy bacillus, but ordinarily bacilli are not found in smears, and only in very small numbers in sections. In occasional cases, however, especially during or after a reaction condition, bacilli can be found in smears and they may be numerous.

Lepromatous. This term signifies of the nature or possessing the qualities of the leproma.

Leprotic and leprous. These terms should be used only in their general sense, signifying pertaining to or affected with leprosy.

Macule. This term is usually applied only to the leprides (neuraltype leprosy), signifying a circumscribed area of skin of abnormal color-varying widely in this character in different races but usually hypopigmented, occasionally hyperpigmented, and of ten erythematous - and commonly with other surface abnormalities, the changes being evident in the whole or only a part of the area. In the terminology of leprosy it is used without regard to the presence or absence of infiltration or elevation. "Macular" is of ten used more generally, in describing lepromatous lesions.

Infiltration. This term is frequently applied in a special sense to a thickening of lepromatous nature which does not have the characters of a papule or nodule, but it is also commonly used in its customary general sense.

Plaque. ()rdinarily this term is applied only to a large leprides in which central resolution is delayed or absent, though it is also used to designate, in a general sense, any large infiltrated area.

Papule. A papule is a small, more or less solid, circumscribed, superficial elevation of the skin, usually but not necessarily circular, conventionally described as varying in size from a pin-head or less to five milimeters in diameter (split-pea size). Papules occur in both forms of leprosy and differ correspondingly in structure and of ten in appearance.

Nodule. A nodule (synonymous with but preferable to "tubercle") is a solid elevation of the skin, of ten similar to a papule except that it is larger; in practice the application of this term is not limited as regards maximum size. Ordinarily it is applied only to lepromata. Nodules are usually more deep-seated than papules, and of ten consist of localized subcutaneous masses.

Polyneuritis. This term has been employed to designate involvement of the main peripheral nerves which results in sensory changes of the extremities that tend to spread centripetally ("acroteric" anesthesia), and in trophic changes of various kinds, and paralyses and atrophies, which may also involve the face. Polyneuritic manifestations do not include the sensory changes in the leprides, or lesions of superficial cutaneous nerves that develop by extension from leprides.

Trophic changes. Under this head are included those changes that are ordinarily ascribed to disturbances of the vasomotor system and of nutrition : anidrosis, glossly skin, ichthyosis, pigmentary changes, loss of hair, perforating ulcers, atrophy and necrosis of bones with consequent mutilations and neuropathic joint lesions. Strictly speaking atrophy and paralysis of muscles. and contractures consequent on them, are not included, but in practice the distinction is seldom made. 


\section{Proposed Revision of the Memorial Conference Classification.}

The following proposed revision of the formula adopted by the Memorial Conference, based on the foregoing considerations, does not lepart essentially from that formula except in so far as is necessary to bring it into line with present knowledge, and in some respects to make it more precise. The primary division into types and the (general) subclassification are retained, but the specifications are elaborated. In addition, however, suggestions-some of them already proposed by other writers-are offered for extending subclassification along other lines, and for indicating certain special features symbolically, but it should be understood that those are secondary matters.

\section{A. Primary classification.}

Neural $(N)$ type. All cases of the "benign" form of leprosy, with disturbances of polyneuritic nature (i.e., peripheral alterations of sensation, trophic disturbances, atrophies and paralyses, and their sequelae), or macular skin lesions (i.e., leprides, usually with localized sensory disturbances), or both, without lepromatous changes in the skin. These cases evidence relative resistance to the infection, are of good prognosis as regards life, and usually react positively to leprolin. The skin lesions are typically though not invariably negative for bacilli, though the nasal mucosa is sometimes positive, and many of them are of tuberculoid nature histologically.

Cutaneous $(C)$ type. All cases of the "malignant" form of leprosy, relatively nonresistant and of poor prognosis, usually negative to leprolin, exhibiting lepromatous lesions of the skin and other organs, especially the nerve trunks. Disturbances of polyneuritic nature may or may not be present; they are usually absent in the earlier stages of primarily cutaneous cases, usually present in the later stages, and of ten present in cases arising secondarily from the neural form.

\section{B. Subclassification.}

The following specifications relate to the unavoidably somewhat crude general subclassification of cases according to the degree of severity or advancement of the disease. They indicate roughly the basis of the division, but in practice it is necessary to consider the entire range of manifestations of a type and endeavour to grade them along the lines indicated into three degrees of advancement.

1. General subclassification, by degree of advancement.

Neural 1 (N1). Slight neural: (a) Cases with from one to several small macules, or a proportionately smaller number of larger ones, whether flat or infiltrated, without indications of polyneuritic changes; or $(b)$ cases presenting only polyneuritic changes of fairly slight degree: peripheral disturbances of sensation affecting one or two extremities, not of marked extent, with only minor trophic disturbances, muscular atrophy or paresis, if any ; or (c) cases showing combinations of macular and polyneuritic manifestations in corresponding degree of total affection. 
Neural 2 (N2). Moderately advanced neural: (a) Cases with fairly numerous or large macules of wide distribution, without evidence of polyneuritic changes or. with such manifestations of fairly slight degree; or $(b)$ cases presenting only polyneuritic changes of moderate degree: peripheral anesthesia of considerable extent if affecting only one extremity, of less extent if multiple; and moderate trophic changes, atrophy and paralyses, including beginning contractures if of limited extent; or $(c)$ cases showing combinations of corresponding total degree.

Neural 3 (N3). Advanced neural: (a) Cases with very numerous or very extensive macular lesions of the most marked kind, with or without polyneuritic changes; or $(b)$ cases presenting only advanced polyneuritic changes : extensive peripheral anesthesia and more or less marked motor and trophic disturbances: paralyses, atrophies, contractures, trophic ulcers and mutilations; or $(c)$ cases showing combinations of corresponding total degree.

Cutaneous 1 (C1). Slight cutaneous : Cases with lepromatous skin lesions consisting of one or a few macular areas, or a few small infiltrated patches or nodules; lesions of mucous membranes are usually absent.

Cutaneous 2 (C2). Moderately advanced cutaneous: Cases with numerous macular areas, or fairly numerous or marked areas of infiltration, or nodules, of lepromatous nature; lesions of mucous membranes are frequently present.

Cutaneous 3 (C3). Advanced cutaneous: Numerous and extensive or very marked lepromatous lesions that may vary as regards stage of development or retrogression, usually with lesions of mucous membranes.

"Mixed" cases. Cases of the cutaneous type usually exhibit, sooner or later, varying degrees of polyneuritic involvement. For precision such "mixed" or "complete" cases may be designated CN. The symbol $\mathrm{C}$ should be given precedence, regardless of the original nature of the case or the relative severity of the two elements, because of the predominant importance of the cutaneous element. In grading the degree of advancement of these cases the appropriate figure is placed after each symbol: e.g., C2-N1, or C1-N3.

Secondary neural cases. Cases that have previously been cutaneous (mixed cases) but in which the lepromatous lesions have resolved leaving symptoms or sequelae of polyneuritic involvement are called "secondary neural."

To effect a balance in grading neural leprosy in the mass, cases in which leprides are a predominant feature should be placed in a given subclass with less advanced polyneuritic manifestations than those cases which present only the latter changes. It is suggested that cases with only macules should not be graded higher than N2 except when those lesions are extensive and of the severe major tuberculoid kind. When macular cases with polyneuritic changes undergo conversion to the cutaneous type, the grading of the $\mathrm{N}$ element will correspond to the degree of the polyneuritic element alone; the skin lesions, having undergone lepromatous transformation, will naturally determine the degree of the $\mathrm{C}$ element.

The division of the cutaneous type indicated refers only to the lepromatous element. If polyneuritic manifestations are present and 
it is desired to indicate their extent, that should be done separately as indicated in the subdivisions of neural leprosy (see "mixed" leprosy).

There is so great a range between the least and the greatest degrees of advancement in leprosy that each of the three gross subdivisions of this classification includes a considerable range within itself. In practice the writer has found it useful to designate by the subgroup symbol, for example N2, those cases that are in about the middle of that range; and, to continue with the same example, indicate by $\mathrm{N} 2-$ and $\mathrm{N} 2+$ those that are less and more advanced-i.e., those that are not much beyond $\mathrm{N} 1$ and those that are approaching N3. This corresponds to the charting on the Wade-le Roux form (39) when the space for each subtype is divided into three.

\section{Special subclassification, by other features.}

The following pertains to subclassification according to special features of the (lisease, especially with regard to the principal varieties. Suggestions for the symbolic representation of other features are offered.

Varieties of cutaneous leprosy. No varieties of cutaneous leprosy have been established that are sufficiently distinct, frequent and general in occurrence to require recognition in formal classification. In places (e.g., India) where many cases show extensive "diffuse" involvement of the skin, not localized in macular areas or infiltrations, there might be an advantage in indicating such cases (as by C.d), but it is not certain that this division would be generally useful.

Principal varieties of neural leprosy. Neural leprosy may be subclassified primarily according to the occurrence of $(a)$ polyneuritic changes and $(b)$ macules, or $(c)$ both. To arrive at a method of indicating these classes it is to be considered that, because $\mathrm{N}$ is the general symbol of the type, it must always be used ( $M$ alone would appear to indicate a third type); and that because of its general significance it would be improper, and also confusing, to use it alone to indicate cases with only polyneuritic manifestations. Two methods seem feasible.

(1) One method presented first because it has been used (MonradKrohn), is to indicate cases with both kinds of changes by NM, those with only polyneuritic manifestations by $N(M)$, and those with only macular lesions-with or without involvement of cutaneous nerves in relation to them-by $(\mathrm{N}) \mathrm{M}$. The degree of severity of advancement of each element could be indicated, when desired, by adding to each symbol a figure corresponding to the degree of the condition as specified in the general subclassification.

For reasons indicated both $\mathrm{N}$ and $\mathrm{M}$ should be used, but with a sign of negation (the parentheses) when one or the other form of change is absent. To use 0 (zero) as proposed by Lie (NOM or NM0) would perhaps invite confusion between it and the letter (O. Positive and negative signs are used for other purposes. With regard to grading, because $\mathrm{M}$ alone would be used simply to indicate the presence of macules, Lie's suggestion that it might indicate the least degree of macular changes would evidently be impracticable.

(2) The above system is open to the serious objection that the use of $\mathrm{N}$ to signify only one element of the neural type would tend 
to fix and perpetuate the existing confusion regarding its proper significance. To avoid that difficulty the following suggestion is offered: $\mathrm{N}$ to be used only to indicate the type, representing its whole concept; NP to indicate neural cases with polyneuritic manifestations but not skin lesions; NM to indicate those with macular changes but not polyneuritic ones; NPM (or NMP) cases with both kinds of changes. Each of the special features could be graded as before.

These symbols, it is suggested, are clear-cut and unmistakable, avoiding any confusion, and are no less simple on the whole than those of the other method.

larieties of maculcs. Classification of cases of the neural type that have macules according to the kinds of those lesions is often desirable in special work, and several writers, including the present one, have offered suggestions for the purpose. There is, however, so much divergence in this matter that, if present knowledge is sufficiently precise to permit making a generally acceptable subdivision of this kind at all, it will probably require action of an international body to do so.

For the symbolic representation of such varieties, it would seem logical to use small letters. Wade and Lie have proposed using " $t$ " to indicate tuberculoid macules. That would be but a beginning in this direction. That symbol seems satisfactory for imprecise use, but it will not suffice if different varieties of that class of lesions are to be indicated. For example, in the writer's classification of macules simple ones could be designated "s," the papulate tuberculoid ones " $p$ " and the minor and major ones perhaps " $t$ " and " $t$ " (the last, italic in printing, to be indicated in writing by an underline).

Indication of the original phase. If it is desired to indicate symbolically in a mixed case the form that occurred first, this can be done readily by placing the prime accent mark ('= primary) after the appropriate letter, as $\mathrm{C}^{\prime} \mathrm{N}$ or $\mathrm{CN}^{\prime}$. This would not interfere with the use of the customary figures to indicate the degree of advancement.

It has been suggested that the distinction in question be made by placing the symbol of the primary form before that of the secondary one (i.e., $\mathrm{CN}$ or $\mathrm{NC}$ ), but that would often subordinate the more important cutaneous phase. Furthermore, Germond's proposal that the symbols be reversed for another purpose complicates the matter. The accent marks are simpler and more obvious of meaning than the "p" which Lie suggests, which might of ten have to follow another small letter, such as "t."

Indication of secondary neural cases. If it should be desired to indicate a secondary neural case, that can be done as $\mathrm{N}^{\prime \prime}("=$ secondary).

Indication of bacteriological status. If for epidemiological or other considerations it is desired to indicate in a case symbol the bacteriological status of a case, that can be done in the way suggested by Lie, by adding $\mathrm{B}+$ or $\mathrm{B}-$ to the case symbol. The $\mathrm{B}+$ would be superfluous in most cutaneous-type cases, and $\mathrm{B}-$ in most neural cases, but $B-$ in a $C$. case would indicate one which has improved to the point indicated, but not enough to be listed as completely arrested or cured, and $\mathrm{B}+$ in a neural case would have obvious significance.

Indication of progression of the disease. If, to obtain a maximum of information in the symbolic representation of a case, it should 
be desired to indicate symbolically whether the disease or any element of it that is indicated in the case-symbol is progressing, retrogressing or stationary, this could be done by placing the acute, grave or circumflex accent, respectively, above the appropriate letter or letters.

Atypical and special cases. There are certain kinds of cases that are not distinguished in the systematic classification. These are: (a) Incipient cases, too little advanced to permit positive identification of its type. For some purposes it is useful to put them in a subclass of their own, but in mass work they are orlinarily put into one or the other types according to the bacteriological findings. (b) Abortive cases, interrupted at an early stage of the disease, usually, if not always, of neural type, and their distinction as a separate group has seldom been made, though it is important from the epiclemiological viewpoint and that of the treating physician. (c) Transitional or intermediate cases, which sometimes give difficulty; this refers chiefly to those which give evidence of changing from the neural type to the cutaneous one. In tuberculoid cases cluring or after reaction the lesions may look as if they had become lepromatous and may be strongly positive bacteriologically, yet their subsequent course may prove that that change had not taken place. Lowe in Calcutta designates such borderline cases "N?C", until the outcome of the condition is determined. Special forms of the disease, such as "lazarine," "bullous," etc., are sometimes clealt with by name but have not found a place in formal classification. 